\title{
Image recognition based on single chip microcomputer processing system research and design
}

\author{
YupingMa \\ ChuanjunWang \\ College of Information Engineering \\ Jilin Engineering Normal University \\ 130052 \\ 65155344@qq.com
}

\begin{abstract}
Keywords: image sensor; Image processing; The man-machine interface
ABSTRACT: This system using the sunplus SPCA561A CMOS image sensor collect the image information, using SPCA563A as image processing chip, image obtained by analyzing and processing the data, and draw the corresponding information, so as to realize the image color, shape recognition, and other functions. To distinguish the results passed to the SPCE061A microprocessor to control the interactive man-machine interface.
\end{abstract}

\section{INTRODUCTION}

Image acquisition is the basis of digital image processing, image recognition, its application field is very broad. Commonly used traditional methods of image acquisition, technology is mature, but there are also some problems. First of all, the output of the CCD camera has been converted into analog NTSC or PAL and mixed with SVideo or video output signal way, this acquisition card sample point on the output sequence to the camera pixel point one to one correspondence, after digital video image quality loss is bigger, the image resolution is also restricted by system. Secondly, the hardware circuit of this method is complex, high cost, is not conducive to promote and popularize use.

Image recognition technology at present in people's life, more and more widely used, such as we are familiar with the digital camera, which has the function of video phone, and we saw some of the smart toys has the function of image recognition. With the continuous development and improvement of image recognition technology, its application in the production and living is becoming more and more widely. For example, the computer image generation, image transmission and communication, high definition television, robot vision and image measurement, image tracking and optical guidance, medical imaging equipment, and so on. Based on this, design a image recognition module, the module can identify the color, shape; Identify location; And the color, shape, location of object to make a simple processing, etc. Mainly used in interactive intelligent toys, image processing products, and in teaching. This module interface is simple, convenient application, easy to combine with MCU, if from sunplus SPCE061A is united in wedlock, make its appearance and, more can reflect the effects of product function.

Image recognition module consists of optical lens, CMOS sensor (SPCA561A), image processing chip (SPCA563A), as shown in figure 1. 


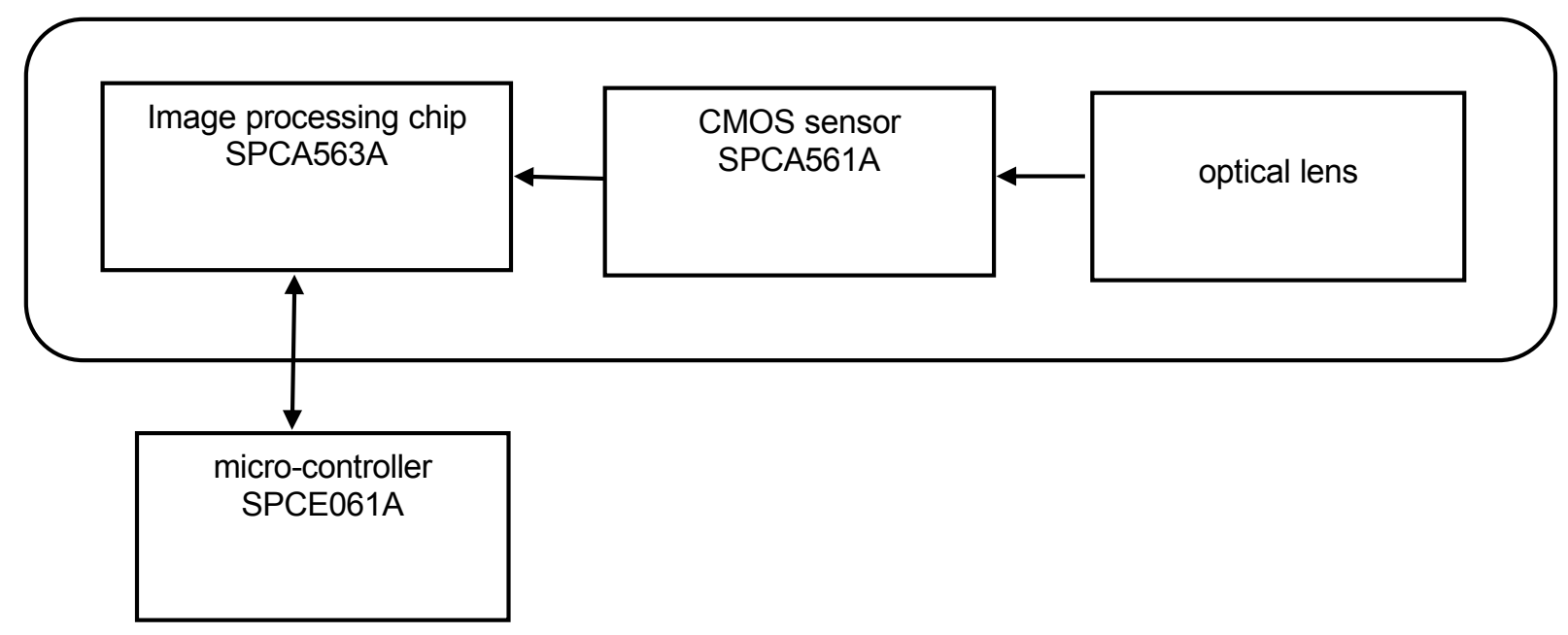

Figure 1. Image recognition module structure diagram

SPCA563A analysis and treatment SPCA561A coming image signal, and draw the corresponding information (color, shape, etc.). The application of module interface is simple, convenient, easy to combine with MCU using image recognition processing system is the concrete application module combined with SPCE061A.

Image recognition module and interface six stitches of the outside world, in turn, meet in SPCA563A VCC, SCK, SD, 3 _reset \&gnd RDY, image recognition. Here, use the SPCE061A sunplus company as the processing chip.

\section{FIRST,OPTICAL LENS}

Generally referred to as the cameras or photography optical lens, its function is optical imaging. Lens is an important component in the machine vision system, has a key role on the imaging quality and its imaging quality of a few the most key indicators have influence, including: resolution, contrast, depth of field and all kinds of aberration. From the focal length optical lens, coke can be divided into short focal lens, telephoto lens; From the view points have wide Angle, standard size, telephoto lens. Structure points have a fixed spot light all prime lens, manual spot light all prime lens, automatic spot light all prime lens, manual zoom lens, automatic zoom lens, automatic aperture electric zoom lens, electric three variable (aperture, focal length, focusing on both variable) lens, etc.

Lens focal length is different, intake of different imaging features, its shape function are different. Different focal length of lens imaging characteristics for photographers depict characters, depicting the environment, foil atmosphere, movement, to grasp the rhythm, such as molding processing, providing favorable conditions.

\section{SECOND ,THE CMOS SENSOR}

CMOS image sensor is developed in recent years a new type of solid-state image sensor, with the same CMOS process, thus can be pixel array and the drive circuit and signal processing circuits are integrated on the same chip. And, now more and more integrate A/D CMOS image sensor chip, so in addition to the analog video output, also can be directly output digital video signal and synchronous signals. Using a CMOS image sensor image acquisition system, the traditional image of A/D acquisition card don't need to, and only need to design appropriate interface circuit. Because of the larger amount of image data, must choose to transmit high speed data interfaces. PC parallel port data transfer rate of up to $2 \mathrm{MB} / \mathrm{s}$, using and programming control and convenient, as image acquisition interface is appropriate. 
CMOS image sensor image signal has the following reading pattern: progressive-scan read out the whole array is a common readout mode; Window read mode read only interested in window like yuan image information, the readout mode increased the interest within the window signal readout rate; Jumping readout mode every one (or two or more) as yuan read image signal, the readout mode with lower resolution image as a cost, to increase the rate of image signal readout. Jumping readout mode combined with window read mode, which can realize digital panoramic camera, tilt camera and zoom camera, etc. CMOS.

Sunplus SPCA561A is a very distinctive chip, without external CMOS sensor, its own 100000 pixels

\section{THIRD,IMAGE PROCESSING CHIP}

Image recognition module uses the image recognition controller is the sunplus SPCA563A. Mainly used in the chip which has the function of image recognition process of interactive intelligent toys, chip built-in the AE/AWB, able to handle the data from the CMOS sensor into QVGA/CIF format. Its internal mainly embedded image capture unit, unit, feature recognition unSP kernel of 16-bit CPU units, ROM unit, etc., has the color recognition, shape recognition, and other functions. Users will be able to use these identification result to control some interactive man-machine interface.

SPCA563A including 16 unSP kernel, CDSP, characteristics of CPU registers unit, sensor interfaces, PLL, serial data interface unit etc. Chip inside with $2 \mathrm{k}$ Word of RAM, $16 \mathrm{k}$ Word ROM.

Characteristics of the processing unit has two important functions: the classification of the target object, image decomposition function. Image decomposition results will register goes into characteristics, external Host CPU can be gained through the way of reading the contents of the register features; Target classification of data stored in the graphics storage register, SPCA563A internal CPU can directly get the classification of the target object information from here and brightness information.

Color segmentation can be finished in this unit to preset decomposition of 7 kinds of color, the register address is $0 \times 7405$. Including the establishment of the logo Can choose is 7 kind of object or 21 kinds of decomposition, it is $0 \times 7404$ register unit. Here basically can get the color, size, space location; If you choose to break down seven kinds of objects that can also get the shape and other information.

Image obtained by the chip of CMOS Sensor information has the function of decomposition and need the contents of the storage to the corresponding address, outside of the Host CPU registers can be read by image features of content to obtain data; SPCA563A internal structure of interface with GLOBAL, make it

expand external ROM, RAM is very easy, can make SPCA563A through specialized USB module, data communication with PC directly.

\section{FOURTH,THE SPCE061A MICRO-CONTROLLER}

SPCE061A sunplus technology research and development production of high performance of a 16 bit single chip microcomputer, it can realize the complex data processing, including basic addition and subtraction and complex product processing. The chip has eight road 10 precision ADC, audio conversion channels of one and the other 7 road can be used as ordinary AD conversion channel. The micro controller not only can realize the basic control function, but also has some DSP function. The chip embedded $32 \mathrm{k}$ FLASH ROM and SRAM, $2 \mathrm{k}$ word integration ADC and DAC, AGC amplifier circuit, etc., is a powerful SOC chip, in order to deal with complex arithmetic also added hardware multiplier, ability of inner product operation item n. $49 \mathrm{MHZ}$ system clock can achieve highest, higher processing speed the SPCE061A can quickly and complex arithmetic logic operation is very easy, is a kind of data acquisition system design is better choice.

Sunplus 16 bit single chip microcomputer has to learn and easy to use the high efficiency of a set of instruction system and integrated development environment. In this environment, support the standard C language, $\mathrm{C}$ language can be calling each other with sunplus assembly language, and provide voice 
playback library function, as long as know the use of the library function, can easily complete the audio playback, which provides convenient conditions to the development of the software.

The SPCE061A chip also integrates a ICE simulation circuit (online) interface, makes the programming, the simulation of the chip is very convenient, and do not take up on the chip ICE interface hardware resources, combination of sunplus provides an integrated development environment ( $\mu$ 'nSP IDE), can use it to real simulation of the chip, and the program's download (burning) is through the interface.

\section{REFERENCES}

1. wangzhaoan, huangjun Editor in chief, power electronic technology, mechanical industry publishing house, 2004.

2. Wang Wujiang. Commonly used integrated circuit quick manual. Beijing: metallurgical industry press, 2004.12.

3. xin-min pan Wang Yanfang compiled, micro computer control technology, the electronic industrial press, in January 2003, the first edition

4. Li Guangdi Zhu Yuexiu Xiu-shan wang By single chip microcomputer based, Beijing university of aeronautics and astronautics press, July 2001

5. Wu Zhongzhi $\mathrm{Wu}$ Jialin compiled, MCU application manuals, mechanical industry publishing house, in April 2003, the second edition 